\title{
ENUCLEACIÓN Y EVISCERACIÓN: ESTUDIO DE 370 CASOS. RESULTADOS Y COMPLICACIONES
}

\section{ENUCLEATION AND EVISCERATION: 370 CASES REVIEW. RESULTS AND COMPLICATIONS}

\author{
VITTORINO M ${ }^{1}$, SERRANO F ${ }^{1}$, SUÁREZ F ${ }^{1}$
}

\section{RESUMEN}

Objetivo: Describir el análisis del resultado quirúrgico de las enucleaciones o evisceraciones realizadas en 370 pacientes en nuestro centro durante 11 años (1990-2000). Se estudió el tipo de corrección realizada y las complicaciones asociadas al procedimiento quirúrgico.

Métodos: Estudio retrospectivo de las historias clínicas de los pacientes que fueron sometidos a los procedimientos mencionados. El cirujano (FS) fue siempre el mismo. Se consideraron los datos demográficos, el diagnóstico, la cirugía ocular previa y asociada, las características de los implantes o injertos, el seguimiento y las complicaciones postoperatorias.

Resultados: Se realizaron 161 enucleaciones y 209 evisceraciones. El 98,4\% de estos procedimientos se asoció a la colocación de algún tipo de implante o injerto. Durante los primeros 6 años del estudio se colocaron injertos lipodérmicos en el $58,8 \%$ de las operaciones realizadas. En los últimos 5 años, el implante de hidroxiapatita constituyó el 90,9\% de los casos intervenidos. En 39 de los 369 pacientes $(10,6 \%)$ se observaron 58 complicaciones, las cuales requirieron algún tipo de cirugía.

Conclusiones: Los implantes de hidroxiapatita consiguieron una excelente reconstrucción de la órbita anoftálmica, y mejoraron los resultados esté-

\begin{abstract}
Purpose: To analyze the surgical results in 370 patients who underwent enucleation or evisceration at our center, during a period of 11 years (19902000), determining the kind of correction used and the complications associated with the procedure.

Methods: This was a retrospective study of medical records from all patients who underwent surgery by the same surgeon (FS) in the Oculoplastic Department. Demographic data, diagnosis, previous and associated ophthalmic surgeries, implant or graft characteristics, follow up period and postoperative complications were determined in all patients.

Results: One hundred sixty-one enucleations and 209 eviscerations were performed. Some kind of implant or graft was associated with $98.4 \%$ of these procedures. During the first 6 years of the study, lipodermal grafts were performed in $58.8 \%$ of the total, while in the last 5 years hydroxyapatite implants were performed in $90.9 \%$ of the cases. In $39(10.6 \%)$ of 369 patients with registered follow up data, 58 complications requiring surgical correction were observed.

Conclusions: Hydroxyapatite implants gave an excellent anophthalmic socket reconstruction, and improved esthetic and motility results. Lipodermal grafts were an excellent alternative in our environment. We observed some complications with the
\end{abstract}

Recibido: 11/5/06. Aceptado: 19/6/07.

Instituto Barraquer de América, Escuela Superior de Oftalmología. Bogotá, Colombia.

1 Doctor en Medicina, Oftalmólogo.

Correspondencia:

Universidad del Rosario

Facultad de Medicina

Calle 13 N. ${ }^{\circ}$ 5-83 Bogotá, Colombia

Telf.: (571) 3474570 
ticos y de motilidad. En nuestro medio los injertos lipodérmicos siguen constituyendo una buena alternativa. Aunque se observaron algunas complicaciones con las diferentes técnicas, muy pocos casos requirieron una nueva intervención quirúrgica.

Palabras claves: enucleación, evisceración, órbita anoftálmica, implantes, hidroxiapatita, injerto lipodérmico. different techniques, but only a few required surgical correction (Arch Soc Esp Oftalmol 2007; 82: 495-500).

Key words: enucleation, evisceration, anophthalmic cavity, implants, hydroxyapatite, lipodermal graft.

\section{INTRODUCCIÓN}

La enucleación del globo ocular fue descrita por primera vez por Bartish en $1583(1,2)$. La técnica que aún se sigue usando fue descrita por Farrell y Bonnet en 1885. En el mismo año Mules publicó el uso del primer implante orbitario posterior a una evisceración (3). Desde hace más de 100 años existe controversia respecto a la elección entre enucleación o evisceración, dependiendo ésta finalmente del origen de la enfermedad (4).

Se han empleado diferentes materiales para implantes, pudiéndose fijar los músculos extraoculares a algunos de ellos (5). En los últimos años, los implantes esféricos porosos (polietileno poroso e hidroxiapatita) son los más utilizados (6), gracias a ventajas como: biocompatibilidad, integración, menor porcentaje de extrusión y menos incidencia de infecciones secundarias (4,7-10).

El injerto lipodérmico es una alternativa en caso de no contar con implantes de otro tipo o para el tratamiento de implantes extruidos. Es un autoinjerto que requiere una técnica quirúrgica meticulosa y un apropiado cuidado postoperatorio (11-13).

\section{SUJETOS, MATERIAL Y MÉTODOS}

Se realizó una revisión retrospectiva de las historias clínicas en 370 pacientes que de manera consecutiva fueron sometidos a evisceración o enucleación en nuestro centro desde el 1 de enero de 1990 hasta el 31 de diciembre de 2000, por uno de los cirujanos del Departamento de Oculoplástica (FS). Se tuvo en cuenta los tipos de implantes utilizados y las complicaciones asociadas. El análisis estadístico se realizó con Excel (Microsoft Corp, Seattle, WA, EEUU).
Se realizaron 161 enucleaciones y 209 evisceraciones. La edad media al momento de la cirugía fue de 31,2 años (rango: 3 meses-86 años), 47 pacientes $(12,7 \%)$ tenían menos de 6 años. El seguimiento medio fue de 36,9 meses (rango 15 días-13,9 años). 228 (61,6\%) pacientes eran hombres y 142 $(38,4 \%)$ mujeres.

Las indicaciones más frecuentes para llevar a cabo el procedimiento fueron traumatismos, glaucomas y neoplasias (tabla I). En 156 ojos (42,3\%) se encontró ptisis bulbi o preptisis de diversas causas. Destacan las secundarias a traumatismos, 77 ojos $(53,2 \%)$, y complicaciones quirúrgicas, 22 casos $(14,7 \%)$. De los 214 pacientes restantes las indicaciones más frecuentes fueron secuelas de traumatismos en 105 ojos (28,5\%), y tumores en 31 casos (8,4\%). El melanoma de coroides, con 9 casos, y retinoblastoma, con 18 casos, constituyeron los más frecuentes en esta categoría. El glaucoma absoluto y buftalmos fueron la causa en 26 casos $(7 \%)$.

El tipo de implante utilizado en esta serie (tabla II) incluyó: injerto lipodérmico en 126 (34,1\%), implantes de nylon (Oertli) 93 (25,2\%) e hidroxiapatita $142(38,5 \%)$. Los restantes 8 casos (2,2\%) estaban constituidos por combinación de las anteriores clases de implantes en 3 pacientes (por

Tabla I. Indicaciones

\begin{tabular}{lcc}
\hline Indicación & $\begin{array}{c}\text { N. } \\
\text { pacientes }\end{array}$ & $\begin{array}{c}\text { Porcentaje } \\
(\%)\end{array}$ \\
\hline Traumatismos & 182 & 49,19 \\
Glaucoma y buftalmos & 33 & 8,92 \\
Tumores & 31 & 8,38 \\
Complicaciones postquirúrgicas & 27 & 7,30 \\
Microftalmos & 19 & 5,14 \\
Otras & 78 & 21,08 \\
\hline \hline
\end{tabular}


insuficiencia del volumen de reconstrucción con el implante inicial), mechas en 2 pacientes con proceso infeccioso orbitario, y ninguno en 3 (debido a infiltración neoplásica orbitaria). Durante los primeros 6 años de este estudio los injertos lipodérmicos correspondieron a 58,8\% de las técnicas empleadas, mientras que en los últimos 5 años el implante de hidroxiapatita constituyó el 90,9\%.

En 50 (94,3\%) de las enucleaciones con implantes de hidroxiapatita se usó recubrimiento escleral del mismo. En las enucleaciones con implante de Oertli se utilizó recubrimiento escleral en solo 6 casos (10\%). En los restantes no se colocó recubrimiento.

La moda del tamaño del implante fue $16 \mathrm{~mm}$ (rango: 14-20 mm), mientras que la colocación de un vástago se realizó en solo 5 pacientes, por preferencia del cirujano.

La enucleación se realizó haciendo una peritomía de la conjuntiva y de la Tenon de $360^{\circ}$. Se individualizan los músculos rectos con gancho de estrabismo, haciendo disección de la Tenon adyacente y reparándolos con sutura doble armada. Se seccionan de las inserciones al globo ocular. Posteriormente se realiza una sección del nervio óptico lo más profundo posible dentro de la órbita con tijeras de enucleación. Si el implante es poroso se cubre con esclera de banco y esta se cierra con puntos separados en «U» de Poliester 5-0 con los nudos intraesclerales, practicándole luego cuatro ventanas a la esclera para imbricar los músculos rectos dentro de las mismas y facilitar la neovascularización del implante. La cápsula de Tenon y la conjuntiva se cierran por planos con puntos separados en «U» (con Poliglactina 6-0).

En las evisceraciones, se realiza una peritomía de $360^{\circ}$, esclerotomía límbica y queratectomía completa con tijeras corneo-esclerales. Se realiza la remoción de todo el tejido uveal con espátula, cucharilla y torundas de gasa. Se introduce el implante o el injerto lipodérmico dentro del casquete escleral. En este último, se le pueden realizar a la esclera cortes de relajación con el fin de ampliar el continente.

El injerto lipodérmico se tomó siempre de la región glútea superior derecha con un trépano diseñado para tal efecto. La técnica utilizada para su implantación en la cavidad orbitaria es similar a la descrita con los implantes en evisceración o enucleación, pero el botón de dermis obtenido se fija en el primer caso al rodete escleral, o en el segundo a los músculos directamente.
Tabla II. Tipo de implante según el procedimiento

\begin{tabular}{lcc}
\hline Tipo de implante & Enucleación & Evisceración \\
\hline Hidroxiapatita & 53 & 90 \\
Injerto lipodérmico & 46 & 80 \\
Oertli & 59 & 34 \\
Otros & 3 & 5 \\
\hline \hline
\end{tabular}

Al terminar se colocó un conformador plástico en la cavidad conjuntival y un vendaje compresivo.

En el postoperatorio se programaron controles cada 3 días en las dos primeras semanas. Posteriormente los pacientes fueron remitidos al ocularista para adaptación de la prótesis ocular cosmética. La movilidad y apariencia estética fueron determinadas subjetivamente por el examinador mediante la simetría respecto al ojo contralateral y el contorno de los párpados.

\section{RESULTADOS}

Los controles postoperatorios se registraron durante un promedio de 36,9 meses (rango 15 días13,9 años). Solamente un paciente no tuvo registro de control postoperatorio.

Se observaron algunas complicaciones que requirieron tratamiento quirúrgico para su corrección en $39(10,6 \%)$ pacientes, 17 en el grupo de pacientes intervenidos de enucleación y 22 en los eviscerados (tabla III). El grupo total estaba formado por 13 (33,3\%) mujeres y 26 (66,7\%) hombres. Estas complicaciones se presentaron desde los 6 días hasta los 10,3 años después de la cirugía (moda: 2 semanas, media: 103 semanas). El rango de edad fue desde 10 meses hasta 79 años (media 29,6 años); del total de pacientes con complicaciones, $11(28,2 \%)$ eran menores de 6 años. Los diversos tipos de implantes presentaron complicaciones que requirieron tratamiento quirúrgico en un porcentaje similar (tabla IV).

En los casos de injerto lipodérmico, llama la atención la aparición de 2 complicaciones que no se presentaron con los otros tipos de implante en nuestra serie: un caso de infección del injerto (resuelto con tratamiento médico antibiótico) y un caso de apertura de la herida glútea que requirió volver a suturarla.

En total se encontraron 14 (3,8\%) casos de exposición del implante o injerto (6 en menores de 6 años), de los cuales 12 fueron sometidos a cirugía, 
Tabla III. Complicaciones que requirieron reintervención según tipo de cirugía e implante

\begin{tabular}{|c|c|c|c|c|c|c|c|c|}
\hline \multirow{2}{*}{ Complicación } & \multicolumn{4}{|c|}{ Enucleación } & \multicolumn{4}{|c|}{ Evisceración } \\
\hline & HA & LD & $\mathrm{OE}$ & Total & HA & LD & $\mathrm{OE}$ & Total \\
\hline Exposición & 6 & - & 1 & 7 & 5 & - & 2 & 7 \\
\hline Extrusión & 1 & - & 1 & 2 & 1 & - & - & 1 \\
\hline Dehiscencia conjuntival & - & 2 & 1 & 3 & - & 2 & 1 & 3 \\
\hline Ptosis palpebral & - & 1 & - & 1 & 2 & 2 & - & 4 \\
\hline Enoftalmos & - & 1 & 1 & 2 & - & 1 & - & 1 \\
\hline Otras & - & 2 & - & 2 & 1 & 3 & 2 & 6 \\
\hline Total & 7 & 6 & 4 & 17 & 9 & 8 & 5 & 22 \\
\hline
\end{tabular}

$\mathrm{HA}=$ hidroxiapatita, $\mathrm{LD}=$ injerto lipodérmico, $\mathrm{OE}=$ Oertli.

Tabla IV. Implantes y complicaciones

\begin{tabular}{lcc}
\hline Tipo de implante & $\begin{array}{c}\mathbf{N}^{\mathbf{o}} \text { de } \\
\text { pacientes } \\
\text { revisados }\end{array}$ & $\begin{array}{c}\mathbf{N} .^{\mathbf{0}}(\mathbf{\%}) \\
\text { complicaciones } \\
\text { quirúrgicas }\end{array}$ \\
\hline Hidroxiapatita & 143 & $17(11,9)$ \\
Injerto lipodérmico & 127 & $14(11)$ \\
Oertli & 93 & $8(8,6)$ \\
\hline \hline
\end{tabular}

mientras que 3 casos $(0,8 \%)$ sufrieron extrusión del implante. El tiempo medio de aparición de exposición fue 58 semanas (rango: 1 a 301 semanas) y 121 semanas (rango 2 a 430 semanas) para la extrusión (tabla V).

En 5 casos $(1,4 \%)$ se observó ptosis palpebral secundaria con indicación quirúrgica. Se realizó la

Tabla V. Cirugía y edad de los pacientes con exposición o extrusión del implante

\begin{tabular}{llccc}
\hline Complicación & Cirugía & $\begin{array}{c}\text { Edad } \\
\text { (años) }\end{array}$ & $\begin{array}{c}\text { Tipo de } \\
\text { implante }\end{array}$ & $\begin{array}{c}\text { Tiempo } \\
\text { exposición } \\
\text { (sem.) }\end{array}$ \\
\hline Exposición & Enucleación & 19 & HA & 1 \\
Exposición & Enucleación & 42 & HA & 2 \\
Exposición & Evisceración & 66 & HA & 2 \\
Exposición & Enucleación & 53 & HA & 2 \\
Exposición & Evisceración & 3 & HA & 5 \\
Exposición & Enucleación & 2 & HA & 5 \\
Exposición & Enucleación & 0,9 & HA & 6 \\
Exposición & Evisceración & 41 & OERTLI & 8 \\
Exposición & Evisceración & 9 & HA & 32 \\
Exposición & Evisceración & 33 & HA & 36 \\
Exposición & Enucleación & 2 & OERTLI & 38 \\
Exposición & Enucleación & 27 & HA & 141 \\
Exposición & Evisceración & 5 & HA & 233 \\
Exposición & Evisceración & 4 & OERTLI & 301 \\
Extrusión & Enucleación & 29 & HA & 2 \\
Extrusión & Evisceración & 3 & HA & 60 \\
Extrusión & Enucleación & 0,9 & OERTLI & 430 \\
\hline \hline
\end{tabular}

$\mathrm{HA}=$ hidroxiapatita. cirugía de ptosis en 3 pacientes (Fasanella-Servat en 1 caso y reinserción de la aponeurosis en 2 casos).

No se encontraron complicaciones en los pacientes a los cuales se les implantó un vástago que requiriesen reintervención quirúrgica.

Los pacientes se enviaron al protésico entre la segunda y la cuarta semanas postoperatorias. En todos los pacientes se observó una adecuada movilidad y una buena adaptación cosmética.

\section{DISCUSIÓN}

Un implante orbitario ideal debe brindar una adecuada movilidad, buen resultado estético y pocas complicaciones. Muchos autores $(4,7,9,14)$ han sugerido que un implante que esté completamente integrado en la órbita, minimizará la posibilidad de migración y de extrusión. El implante microporoso de hidroxiapatita reúne estos requisitos y por lo tanto se convirtió en el más usado (7). Otros implantes como el de Oertli o el injerto lipodérmico fueron usados también en la corrección de la órbita anoftálmica, especialmente en los primeros años de la década del 90. Es importante resaltar que el injerto lipodérmico sigue constituyendo una excelente alternativa como implante intraorbitario, sobre todo en lugares donde la condición económica del paciente es un factor determinante.

La mayoría de las complicaciones secundarias a estas cirugías se pueden resolver adecuadamente como se demuestra en el presente estudio. No hubo diferencia significativa de la tasa de complicaciones entre los diferentes tipos de implantes utilizados. Las complicaciones en nuestra serie fueron menores que las publicadas en la literatura mundial $(15,16)$, requiriendo cirugía para corregirlas en pocos casos. Varios estudios han revisado el uso de implantes 
porosos y sus complicaciones $(8,17-23)$. Los diferentes rangos de exposición de implantes de hidroxiapatita van desde 0 a $22 \%(16,18,19,24-27)$.

A 5 implantes de hidroxiapatita les fue colocado un vástago, número que al ser tan pequeño no nos permite un análisis estadístico significativo relacionado con este tipo de accesorio. Sin embargo se han descrito diversas complicaciones (25). La tendencia es a no seguir utilizándolo por los daños que causa en el implante con los movimientos oculares extremos.

La apariencia final estética y el movimiento de la prótesis fue considerada adecuada por el cirujano en los 369 pacientes que tuvieron seguimiento, coincidiendo con otras citas de la literatura $(7,24)$.

La edad de los pacientes podría influir ya que observamos una clara tendencia a tener exposición o extrusión del injerto en los pacientes de edad pediátrica (23). Una de nuestras hipótesis es que el frote, más difícil de manejar que en un paciente adulto normal, podría incidir en este resultado.

En la actualidad se cuenta con diversas técnicas de manejo de la órbita anoftálmica, todas ellas buscan un equilibrio entre un buen resultado del complejo implante-prótesis y el menor número de complicaciones. Sin embargo, sólo estudios a largo plazo permitarán elegir la mejor opción para los pacientes.

\section{BIBLIOGRAFÍA}

1. Luce CM. A short history of enucleation. Int Ophthalmol Clin 1970; 10: 681-687.

2. Arruga A. La mutilación deliberada de los ojos. Arch Soc Esp Oftalmol 2003; 78: 339-340.

3. Gougelmann HP. The evolution of the ocular motility implant. Int Ophthalmol Clin 1970; 10: 689-711.

4. Migliori ME. Enucleation versus evisceration. Curr Opin Ophthalmol 2002; 13: 298-302.

5. Moshfeghi DM, Moshfeghi AA, Finger PT. Enucleation. Surv Ophthalmol 2000; 44: 277-301.

6. Su GW, Yen MT. Current trends in managing the anophthalmic socket after primary enucleation and evisceration. Ophthal Plast Reconstr Surg 2004; 20: 274-280.

7. Christmas NJ, Gordon CD, Murray TG, Tse D, Johnson T, Garonzik $S$, et al. Intraorbital implants after enucleation and their complications: a 10-year review. Arch Ophthalmol 1998; 116: 1199-1203.

8. Remulla HD, Rubin PA, Shore JW, Sutula FC, Townsend DJ, Woog JJ, et al. Complications of porous spherical orbital implants. Ophthalmology 1995; 102: 586-593.

9. Custer PL. Enucleation: past, present, and future. Ophthal Plast Reconstr Surg 2000; 16: 316-321.
10. Núñez Sánchez, A, Reche Sainz JA, Sanz López, A, Mateos Sánchez, E, García Llanes G, San Miguel Fraile P, et al. Integración de los implantes orbitarios de polietileno (Medpor). Estudio histológico. Arch Soc Esp Oftalmol 2001; 76: 25-29.

11. Lisman RD. Dermis-fat grafting. In:Della Rocca RC, Nesi FA, Lisman RD. Ophthalmic Plastic and Reconstructive Surgery. St Louis: CV Mosby. 1987: 1308-1320.

12. Shore JW, McCord CD Jr, Bergin DJ, Dittmar SJ, Maiorca JP, Burks WR. Management of complications following dermis-fat grafting for anophthalmic socket reconstruction. Ophthalmology 1985; 92: 1342-1350.

13. Guberina C, Hornblass A, Smith B. Pitfalls of autogenous lipodermal implantation to the orbit. Ophthal Plast Reconstr Surg 1987; 3: 65-70.

14. Beard C. Remarks on historical and newer approaches to orbital implants. Ophthal Plast Reconstruct Surg 1995; 11: 89-90.

15. Hornblass A, Biesman BS, Eviatar JA. Current techniques of enucleation: a survey of 5,439 intraorbital implants and a review of the literature. Ophthal Plast Reconstr Surg 1995; 11: 77-86; discussion 87-88.

16. Kaltreider SA, Newman SA. Prevention and management of complications associated with the hydroxyapatite implant. Ophthal Plast Reconstruct Surg 1996; 12: 18-31.

17. Ashworth JL, Rhatigan M, Sampath R, Brammar R, Sunderland $S$, Leatherbarrow $B$. The hydroxyapatite orbital implant: a prospective study. Eye 1996; 10: 29-37.

18. Shields CL, Shields JA, De Potter P, Singh AD. Problems with the hydroxypatite orbital implant: experience with 250 consecutive cases. Br J Ophthalmol 1994; 78: 702-706.

19. Oestreicher JH, Liu E, Berkowitz. M. Complications of hydroxyapatite orbital implants. A review of 100 consecutive cases and a comparison of Dexon mesh (polyglycolic acid) with scleral wrapping. Ophthalmology 1997; 104: 324-329.

20. Nunery WR, Heinz GW, Bonnin JM, Martin RT, Cepela $M A$. Exposure rate of hydroxyapatite spheres in the anophthalmic socket: histopathologic correlation and comparison with silicone sphere implants. Ophthal Plast Reconstr Surg 1993; 9: 96-104.

21. Ainbinder DJ, Haik BG, Tellado M. Hydroxyapatite orbital implant abscess: histopathologic correlation of an infected implant following evisceration. Ophthal Plast Reconstr Surg 1994; 10: 267-270.

22. Karcioglu ZA, al-Mesfer SA, Mullaney PB. Porous polyethylene orbital implant in patients with retinoblastoma. Ophthalmology 1998; 105: 1311-1316.

23. Li T, Shen J, Duffy MT. Exposure rates of wrapped and unwrapped orbital implants following enucleation. Ophthal Plast Reconstr Surg 2001; 17: 431-435.

24. Shields CL, Shields JA, De Potter P. Hydroxyapatite orbital implant after enucleation. Experience with initial 100 consecutive cases. Arch Ophthalmol 1992; 110: 333-338.

25. Dutton JJ. Coralline hydroxyapatite as an ocular implant. Ophthalmology 1991; 98: 370-377.

26. De Potter P, Shields CL, Shields JA, Singh AD. Use of the hydroxyapatite ocular implant in the pediatric population. Arch Ophthalmol 1994; 112: 208-212.

27. Buettner H, Bartley GB. Tissue breakdown and exposure associated with orbital hydroxyapatite implants. Am J Ophthalmol 1992; 113: 669-673. 